\title{
La science de l'écriture et la linguistique : Benveniste, l'artisan de la (ré)conciliation?
}

\author{
Isabelle Klock-Fontanille ${ }^{1}$ \\ CeReS (Centre de Recherches Sémiotiques), Université de Limoges, Institut Universitaire de France, \\ France
}

\begin{abstract}
Résumé. En 2012, paraît un ouvrage à la fois essentiel et perturbant pour qui s'intéresse à la science de l'écriture: Dernières leçons, Collège de France, 1968 et 1969, dont huit leçons sont consacrées à la question de l'écriture (sur les 16 leçons que comporte le volume). Le contenu de ces leçons était resté inédit jusque là. Au moment où Benveniste rédige ces cours sur l'écriture, la science de l'écriture est à un tournant : à cette époque, elle est dominée par une conception représentative issue de la tradition saussurienne (l'écriture comme code second destiné à représenter la langue). L'heure est à la remise en question, avec, en particulier Derrida. Grâce à un dialogue entre sémiotique, anthropologie et une conception revisitée de la linguistique, la science de l'écriture se trouve peu à peu profondément renouvelée. Les réflexions de Benveniste ont-elles contribué à ce renouveau de la science de l'écriture ? Et plus précisément, ses propositions ont-elles contribué à renouveler l'approche dite linguistique ? Nous essayerons de comprendre quelles implications ses réflexions ont eues, mais aussi quelles en sont les limites. Nous terminerons par des pistes pour un point de vue global sur le problème.
\end{abstract}

\begin{abstract}
The science of writing and linguistics. Benveniste, the architect of (re) conciliation? In 2012, a work that is both essential and disturbing for anyone interested in the science of writing appears: Dernières leçons, Collège de France, 1968 et 1969, of which eight lessons are devoted to the question of writing (of the 16 lessons in the volume). The content of these lessons had remained unpublished until then. At the time when Benveniste wrote these courses on writing, the science of writing was at a turning point: at that time, it was dominated by a representative conception coming from the Saussurian legacy (writing as a second code intended to represent the language). Now is the time to question, with, in particular, Derrida. Thanks to a dialogue between semiotics, anthropology and a revisited conception of linguistics, the science of writing is gradually being renewed. Did Benveniste's reflections contribute to this revival of the science of writing? And more specifically, did his proposals contribute to renewing the so-called linguistic approach? We will try to understand what
\end{abstract}

${ }^{1}$ isabelle.klock-fontanille@unilim.fr 
implications his reflections have had, but also what are their limits. We will end with tracks for a global point of view on the problem.

\section{Introduction}

En 2012, paraît un ouvrage à la fois essentiel et perturbant pour qui s'intéresse à la science de l'écriture : Dernières leçons, Collège de France, 1968 et 1969 (EHESS/Seuil/Gallimard, 2012). Jean-Claude Coquet et Irène Fenoglio ont publié les derniers cours de linguistique générale de Benveniste, dont huit leçons sont consacrées à la question de l'écriture (sur les 16 leçons que comporte le volume). Le contenu de ces leçons était resté inédit jusque là. Cette publication est l'aboutissement d'un long travail pendant lequel les deux auteurs ont rassemblé les manuscrits, les ont transcrits, édités.

Benveniste a traité le problème de l'écriture tardivement dans sa carrière, en 1968-1969, et il faudra attendre 2012 pour que ces textes soient publiés. Dans l'introduction au volume, Jean-Claude Coquet et Irène Fenoglio rappellent que ce thème a souvent été annoncé dans les articles de linguistique générale mais jamais traité pour lui-même $(2012,42)$. En effet, dans l'article "Sémiologie de la langue » qu'on trouve dans les Problèmes de linguistique II, mais qui était paru à l'origine dans Semiotica en 1969, on peut lire : « De l'écriture nous ne dirons rien ici, réservant pour un examen particulier ce problème difficile » (Benveniste 1974, 50). Mais comme le rappelle J.-Cl. Coquet, « l'écriture est bien ce qui nous est apparu comme le point nodal de la recherche à la fin de sa vie » $(2016,59)$.

$\mathrm{Au}$ moment où Benveniste rédige ces cours sur l'écriture, la science de l'écriture, qui a connu bien des vicissitudes, est à un tournant : à cette époque, l'écriture est souvent réduite à une fonction ancillaire (un système second destiné à représenter la langue, selon Saussure, une « sémie substitutive », selon les termes de Buyssens [1943, 49]). L'heure est à la remise en question. Il y a une dévalorisation de l'approche linguistique qu'on réduit - à tort - à cette fonction ancillaire de l'écriture. La science de l'écriture, après avoir été, comme il a été souvent dit, " le parent pauvre de la linguistique », a été l'objet d'un renouvellement grâce à la sémiotique et l'anthropologie. Mais depuis un certain nombre d'années, une approche linguistique revisitée permet de repenser la science de l'écriture, sous l'impulsion, en particulier, de linguistes comme Roy Harris (Klock-Fontanille 2019). Ces écrits de Benveniste sur l'écriture (même si tout n'est pas clair, en raison même de leur caractère inachevé, non stabilisé) participent à ce renouveau dans la réflexion sur cette science de l'écriture, toujours en débat actuellement. Et même, ils proposent une approche linguistique très différente de celle d'un Roy Harris, par exemple.

\section{Les vicissitudes de la science de l'écriture.}

D'Aristote à Saussure en passant par Platon et d'autres, la science de l'écriture a semblé ne pas avoir de raison d'exister, puisque l'écriture elle-même n'avait pas de statut indépendant et autonome.

Rappelons ces citations célèbres, toujours présentées lorsqu'on veut mettre en évidence ce statut inférieur de l'écriture. Pour Aristote, « les mots prononcés sont des symboles ou des signes des affections ou des impressions de l'âme; les mots écrits sont des signes des mots prononcés. » (De Interpretatione, I, 4-6). Quant à Saussure, il affirme que :

Langue et écriture sont deux systèmes de signes distincts ; l'unique raison d'être du second est de représenter le premier; l'objet linguistique n'est pas défini par la 
combinaison du mot écrit et du mot parlé ; ce dernier constitue à lui seul cet objet. Mais le mot écrit se mêle si intimement au mot parlé dont il est l'image, qu'il finit par usurper le rôle principal ; on en vient à donner autant et plus d'importance à la représentation du signe vocal qu'à ce signe lui-même. C'est comme si l'on croyait que, pour connaître quelqu'un, il vaut mieux regarder sa photographie que son visage.

(Saussure 1986, 45)

De nombreuses études existent sur la conception de l'écriture chez Saussure. Chez lui, l'écriture est ambivalente. Et comme le rappelle P.-Y. Testenoire, «l'écriture pose littéralement problème au linguiste en ce qu'il la considère comme un système extérieur à la langue mais intégré à celle-ci » (Testenoire 2017, 117).

Dans le chapitre 6 du $C L G$ intitulé « Représentation de langue par l'écriture », dont la citation ci-dessus est extraite, l'écriture est dévalorisée. Elle a un rôle secondaire. Il y a chez Saussure une primauté de l'oral. Dès les premières pages du $C L G$, Saussure s'évertue à nous mettre en garde contre cette «maitresse d'erreur et de fausseté », qu'est l'écriture, afin que les linguistes ne détournent aucune de leurs pensées de l'objet véritable à décrire, qui est la langue, la langue orale ou phonémique, c'est-à-dire qui passe par le phonème. On peut dire que, en mettant la théorie phonologie au centre de la linguistique, cette tradition issue de Saussure a exclu l'écriture.

C'est donc une vision représentative de l'écriture que Saussure propose. J.-L. Chiss et Ch. Puech le rappellent :

On sait aussi - en particulier depuis les analyses de J. Derrida (1967) -, combien le terme de « représentation » est central dans le $C L G$ et combien le champ sémantique dans lequel il s'insère est lourd de connotations dévalorisantes dès lors qu'il est question de l'écriture : « usurpation », « travestissement », « illusion », « trahison ». Chiss et Puech 1988, 48)

Cette fonction ancillaire de l'écriture remonte bien loin : nous avons hérité des Grecs ce primat de l'oral. Pour les Grecs, il y a une supériorité évidente de la parole sur l'écriture. Contrairement aux civilisations cunéiforme ou égyptienne, qui l'avaient précédée, la civilisation grecque ne voit dans les signes écrits qu'une peinture appauvrie de la voix. Dans un célèbre passage du Phèdre, relatant le mythe de l'invention de l'écriture par le dieu égyptien Thot (Phèdre, 274-277), Platon a refoulé l'écriture comme responsable de la ruine de la mémoire, et Saussure, qui montre dans le CLG l'importance de la prise de conscience du mode de fonctionnement propre au langage, commence par la dévalorisation de l'écriture, en présentant celle-ci comme la simple reproduction de la parole : "L'écriture voile la vue de la langue : elle n'est pas un vêtement, mais un travestissement » (Saussure 1986, 51). Il y a donc un lien de subordination et de dévalorisation de l'écriture par rapport à la parole chez Saussure. Même si, comme Chiss et Puech l'ont souligné, les choses ne sont pas aussi monolithiques dans le $C L G$ (Chiss et Puech 1988). Les travaux de Testenoire montrent que la question de l'écriture chez Saussure est plus complexe et plus ouverte, mais cela n'apparaît pas si l'on s'en tient au seul Cours de linguistique générale établi par Bally et Sechehaye. L'approfondissement du corpus saussurien permet d'apporter des précisions (Testenoire 2017, 2018). Ajoutons que Saussure construit son objet qui est le système de la langue, dans lequel il n'y a pas d'entrée pour l'écriture, qui, du coup, est renvoyée du côté de la sémiologie.

Quoi qu'il en soit, Saussure, ou du moins la lecture qui en a été faite, est le promoteur de l'approche traditionnelle - dite linguistique - de l'écriture, dont on peut présenter rapidement et schématiquement les deux grands principes qui la fondent (Klock-Fontanille 2016) :

1. Tout d'abord, l'écriture est un code second destiné à représenter la langue (vision représentative). Le fait que nous soyons capables de transcrire à peu près tout ce que nous disons et que tout ce qui est écrit puisse être lu à haute voix nous invite irrésistiblement à conclure que l'écriture n'est que de la parole notée. C'est ce que nous croyons depuis Aristote, et, en passant par Rousseau, c'est ce que nous pouvons lire chez Bloomfield, Saussure et bien d'autres. 
2. Ensuite, c'est l'idée que le système alphabétique d'écriture est techniquement supérieur (vision téléologique). Comparé aux autres systèmes d'écriture, l'alphabet serait le seul à avoir réussi à représenter tout ce qui peut être dit. Il y serait parvenu en transcrivant les constituants sonores élémentaires de la parole, les phonèmes. L'invention de l'alphabet par les Grecs est considérée comme l'un des sommets de l'évolution culturelle. Les grandes théories classiques relatives à l'invention et à l'histoire de l'écriture, celles de Cohen (1958), de Gelb (1952), de Février (1959) et de Diringer (1968), ont en commun de considérer l'évolution de l'écriture de la même manière : la représentation des idées au moyen d'images, celle des mots grâce à des signes logographiques, celle des sons grâce à l'invention des syllabaires : autant de tentatives avortées ou de tâtonnements inféconds sur la voie de l'invention de l'alphabet, point culminant de cette recherche, supérieur à tous les autres. Le progrès intellectuel apparaît ainsi lié à l'alphabet. Notons que cette évolution est aussi une typologie.

\section{Derrida : un grand coup de pied dans la fourmilière}

$\mathrm{Au}$ moment où Benveniste réfléchit à la question de l'écriture, l'atmosphère est à un grand coup de pied dans la fourmilière.

Nous sommes en 1969. A cette date, les recherches sur l'écriture sont à la fois dans une impasse et dans un tournant. C'est en effet l'époque des grandes, monumentales, histoires de l'écriture, que nous avons mentionnées : celles de Février, de Cohen et de Gelb. Précisons que ces trois ouvrages ont été inlassablement recopiés, y compris récemment. Comme l'explique Battestini, les histoires de l'écriture, même les plus récentes,

organisent et réduisent à un continuum arbitraire et fictif des faits et événements isolés ou dont les liens ne sont nullement attestés, sans réelle consécutivité ni conséquentialité. L'immense majorité d'entre eux est disséminée dans le temps et dans les espaces géo-culturels. La fiction d'un continuum, toujours invoquée, qui va simplement des pictogrammes à l'alphabet latin, se voit attribuer une valeur universelle. Toujours, l'écrit est entendu comme dénotant la parole. (Battestini 1997, 34-35)

A l'origine de cette histoire sont situées des proto-écritures et pré-écritures, dites «primitives », toutes imprégnées et proches de l'image, à laquelle elles auraient d'abord emprunté leurs attributs. Au point d'aboutissement, la supériorité absolue de l'alphabet des sociétés occidentales, jugé comme une sorte d'idéal insurmontable, et après lequel il n'y a plus rien à dire. Selon cette « histoire », les efforts de l'humanité pour se doter de l'écriture aboutissent, après cinq millénaires, au triomphe d'un système graphique qui a permis le développement littéraire et scientifique de l'Europe et de ses anciens Empires.

Cependant, en 1967, le structuralisme est interpellé par deux parutions de Derrida, à partir du champ de la philosophie : De la grammatologie et L'Ecriture et la Différence, qui paraissent simultanément et qui vont provoquer du bougé dans la structure. Comme l'explique François Dosse, dans son Histoire du structuralisme, Derrida va " entamer un travail de déconstruction systématique de chaque œuvre structuraliste en y repérant autant de traces d'un logocentrisme qui reste à dépasser » (Dosse 1992, 31). Derrida, dont l'ouvrage De la grammatologie paraît en 1967, est allé très loin dans la critique : pour lui, il y a eu un refoulement de l'écriture par la civilisation occidentale au profit de la phonè :

L'Occident depuis Platon privilégie la voix, considérée comme véritable essence, porteuse de sens, aux dépens de l'écriture. Toute la trame historique de l'Occident ne serait que l'histoire de cette évacuation. (Dosse 1992, 39)

Par ailleurs, s'interrogeant sur son objet - l'écriture -, Derrida remarquait :

Moins qu'un autre, le grammatologue peut éviter de s'interroger sur l'essence de son objet sous la forme d'une question d'origine : «Qu'est-ce que l'écriture ? » veut dire « où et quand commence l'écriture ?» Les réponses viennent en général très vite. 
Elles circulent dans des concepts fort peu critiqués et se meuvent dans des évidences qui semblent depuis toujours aller de soi. Autour de ces réponses s'ordonnent chaque fois une typologie et une mise en perspective du devenir des écritures. Tous les ouvrages traitant de l'histoire de l'écriture sont composés selon la même forme : une classification de type philosophique et téléologique épuise les problèmes critiques en quelques pages et l'on passe ensuite à l'exposition des faits. Contraste entre la fragilité théorique des reconstructions et la richesse historique, archéologique, ethnologique, philologique de l'information. (Derrida 1967, 44)

Cette question a-t-elle eu une influence sur Benveniste ? Il est difficile de le dire, mais il est certain que cette entreprise de déconstruction va ouvrir des perspectives dans plusieurs directions.

Actuellement, tout le monde est à peu près d'accord pour dire que l'approche linguistique de l'écriture telle qu'elle est traditionnellement présentée n'est plus tenable. Cette volonté de redéfinir le signe écrit et d'autonomiser le scriptural s'est accomplie par l'ouverture à d'autres disciplines, en particulier la sémiotique et l'anthropologie.

Il s'agissait à la fois de réintroduire l'image et d'autonomiser le signe écrit par rapport à l'oral. L'autonomie de l'écriture est revendiquée par ceux qui s'éloignent du point du vue strictement linguistique. S. Battestini est de ceux-là, il est représentatif de l'approche que Klinkenberg appelle " pan-sémiotique » (Klinkenberg 2005, 161). Certes, ce point de vue est celui du savant des écritures africaines. Dans son dernier ouvrage, S. Battestini revendique cette approche : « la linguistique qui avait écarté l'écriture de son objet, se souvient qu'un de ses maîtres parmi les plus influents (Saussure) l'avait confiée à la sémiotique » (Battestini 2006, 5). Pour Battestini, l'écriture et le script ne seront plus seulement considérés sous l'angle de leur rapport à la langue, mais aussi dans leurs relations avec l'image :

La fonction de l'écriture ne peut plus être limitée à la notation de la parole, pas plus à celle indirectement de la pensée, mais est plus généralement perçue de nos jours comme chargée d'archiver et de transmettre de la pensée organisée, du texte. Il ne s'agit donc plus d'écriture au sens ordinaire et ethnocentrique mais d'une technique, celle de l'inscription du sens. (Battestini 2006, 24)

C'est pourquoi, il préfère le terme de «script»: ce terme désigne tous les modes " graphiques » et autres de conservation et de transmission des savoirs endogènes et des messages, dont les écritures phonologiques, synthétiques et analogiques ainsi que les objets et les images dont la fonction sociale peut leur être similaire.

Il paraîtra évident que la sémiotique, selon le vœu de Saussure, prenne ici le relais de la linguistique puisque le «script» est un système de signes, agissant « au sein de la vie sociale ». Les alphabets occupent une place " privilégiée » dans cet ensemble que désigne le mot script, mais dans la catégorie des écritures phonocentriques (consonantiques, syllabiques et alphabétiques), elles-mêmes côtoyées par les écritures synthétiques (un signe dénote un segment discursif ou narratif) et analytiques (un signe dénote une idée, un fait ou un objet). Le concept de script inclut et déborde doublement ce champ traditionnel de la grammatologie et des histoires de l'écriture, puisqu'il fait une place à l'inscription du sens de l'art (objets inscrits, images, signes figuratifs, abstraits et discrets mais tous plus ou moins systématisés ou codifiés) au même titre qu'aux écritures non-phonocentriques comme modes de production, conservation et transmission de la pensée, des savoirs et de la mémoire collective. (Battestini 2006, 24)

Cette conception large a beaucoup d'adeptes. Pour Derrida, il y a écriture dès qu'il y a « inscription durable d'un signe ».

Cette approche très large, «pan-sémiotique » s'oppose au point de vue strictement logocentrique. Klinkenberg le rappelle :

Il faut souligner que les débats sur la relation entre langue écrite et langue orale dissimulent mal une hésitation entre deux conceptions de l'écriture, objet sur la 
définition de quoi il faut pourtant bien s'entendre. Ces deux conceptions, diamétralement opposées, peuvent être appelées conception large, ou pan-sémiotique, et conception stricte, ou logocentrique. (Klinkenberg 2005, 161)

Klinkenberg poursuit :

Pour les tenants de la conception stricte, l'écriture est une " sémie substitutive ", selon l'expression de notre confrère Eric Buyssens (1943 : 49) : elle a pour unique fonction sémiotique de noter le langage oral. [...] Et dès lors, puisqu'aucune autonomie ne lui est reconnue par rapport à la langue orale, toute fonction sémiotique propre lui est déniée.

Remarquons que, trop longtemps prisonnière d'une vision représentative de l'écriture, la recherche, lorsqu'elle s'en est libérée, a eu parfois tendance à oublier une caractéristique intrinsèque de l'écriture. $\mathrm{C}$. Touratier fait deux mises au point sur cette question (Touratier 2004) :

1. Première observation : l'écriture ne fait pas partie de la langue et n'entre pas du tout dans la définition de ce qu'est le langage. L'écrit est second par rapport à l'oral. L'écriture des mots français est un codage pour représenter graphiquement le Sa des morphèmes qui est phonique ;

2. Deuxième observation : l'écriture est indissociable du langage. Certaines écritures notent le Signifiant des énoncés (écritures alphabétiques et syllabiques), soit le Signifié (écritures synthétiques et analytiques).

\section{Le programme de Benveniste : «Ne confondons pas l'écriture avec la langue écrite (je prends cette expression comme signifiant "la langue sous forme écrite") » (Leçon 8, p. 91).}

Voici comment Benveniste résumait cette partie du cours de 1968-1969 portant sur l'écriture: Enfin, nous avons examiné les rapports entre la langue et le système sémiotique constitué par l'écriture. Au terme d'un examen détaillé qui nous a fait parcourir les différents modèles d'écriture attestés dans l'histoire, il nous est apparu que, contrairement à l'idée admise partout, l'écriture ne constitue pas un système distinct. C'est le prolongement ou la projection de la langue même, et donc la même situation à l'égard des systèmes extra-linguistiques. Nous voyons dans l'écriture l'instrument et la manifestation du procès d'auto-sémiotisation de la langue. Un aperçu des résultats esquissés ici sera prochainement publié dans la nouvelle revue Semiotica. (Benveniste 1969, 364)

Ces cours ont été étudiés en détail par plusieurs chercheurs, en particulier I. Fenoglio dans plusieurs articles (Fenoglio 2013, 2016, 2017). Nous retiendrons quelques points qui nous paraissent essentiels dans la réflexion sur la question de la science de l'écriture.

Le point central chez Benveniste est la question de la relation langue-écriture. Les cours portent d'ailleurs le titre de "La langue et l'écriture ». Mais il va repenser le lien. Il s'agit de « repenser à neuf, dans leur relation primordiale, la langue et l'écriture », écrit-il au début de la leçon 8 (p. 91).

Dès le début de ce premier cours consacré à l'écriture, Benveniste à la fois se réfère à Saussure et prend ses distances avec lui, dans la mesure où, dit-il, Saussure «confond l'écriture avec l'alphabet, et la langue avec une langue moderne » (p. 92), et postule que l'écriture est un « système subordonné à la langue » (p. 95). Or, «l'acte d'écrire ne procède pas de la parole prononcée » (p. 94).

Comme le rappelle Testenoire, c'est à une sorte de doxa sur la conception saussurienne de l'écriture que se réfère Benveniste : 
La réflexion benvenistienne sur l'écriture s'appuie sur celle de Saussure pour la contester et la dépasser. Dans le cours de 1968-1969 au Collège de France, qu'ont publié Jean-Claude Coquet et Irène Fenoglio à partir des notes préparatoires du linguiste et des cahiers d'étudiants, la leçon du 3 février 1969 (numérotée 8 dans l'édition) est spécifiquement consacrée à la critique de l'analyse saussurienne de l'écriture. Cette critique se fonde exclusivement sur le Cours de linguistique générale. Le traitement de l'écriture dans le $C L G$ se réduit, rappelons-le, à deux passages, parfois tenus pour contradictoires. Le premier est le chapitre 6 de l'introduction intitulé «Représentation de la langue par l'écriture » où est défendue la thèse selon laquelle l'écriture est un système de représentation trompeur de la langue (Saussure [1916] 1967 : 44-54). Le second passage se trouve dans le chapitre consacré à la valeur où est développée une comparaison entre le signe linguistique et le signe graphique avec l'image des trois $t$ dont les variantes matérielles seraient indifférentes (Saussure [1916] 1967 : 165-166). (Testenoire 2018)

La critique de Benveniste porte sur le phonocentrisme saussurien, c'est-à-dire sur la conception de l'écriture comme une forme seconde et dégradée de la langue. Cependant Benveniste, n'adopte pas une position autonomiste. Son postulat est que l'un des traits définitoires de l'écriture, c'est le lien entre langue et écriture et ce lien est «primordial». Comme l'explique I. Fenoglio,

Le terme « primordial » n'est pas anodin, il n'est pas choisi au hasard et il faut le saisir dans toute sa puissance. Ce terme (du latin primordium : origine, avènement) signifie à la fois essentiel, fondamental, primitif et premier. Par cette insistance, Benveniste indique sa voie et désigne son objet. (Fenoglio 2017, 215)

Cette posture a pour conséquences que :

1. Pour interroger l'acte d'écrire et le comprendre, Benveniste remonte loin, à l'invention de l'écriture. Ce qui l'intéresse, c'est de comprendre le lien exact établi entre la langue et l'écriture en s'interrogeant sur la façon dont elle a été inventée sans toutefois, précise-t-il, rechercher l'« origine de l'écriture »; il cherche à comprendre les diverses solutions de la « représentation graphique » que les hommes ont trouvées pour signifier.

2. Ce lien est essentiel : certes, l'écriture est une « invention », mais elle est en langue.

3. Comment s'établit cette relation? Ce sont les procédures de conversion, de transposition qui vont l'intéresser : il parle à deux reprises de « la conversion soudaine de la langue en image de la langue. » (p. 93). La relation langue-écriture est une relation de conversion réciproque.

4. Mais l'écriture n'est pas subordonnée à la langue, elle n'est pas un simple instrument, l'écriture est un système sémiotique.

Comme le note I. Fenoglio, Benveniste va aborder la question de l'écriture du point de vue du linguiste.

Mais ce point de vue est celui d'un linguiste profondément éclairé d'anthropologie ; un linguiste pour qui le langage sert «non seulement à communiquer mais à vivre ", selon ses propres termes. Il considère donc le phénomène de l'écriture dans une amplitude à la fois spatiale et historique, remontant même à la pré-histoire mais tout en ayant clairement à l'esprit la nature exacte du travail que peut se proposer du linguiste. (Fenoglio 2017, 213)

La question de l'écriture est donc bien un problème théorique pour Benveniste. I. Fenoglio présente le brouillon du plan de cours de Benveniste (Fenoglio 2017, 216) :

Langue et écriture

I Écarter les notions usuelles sur

1) l'écriture représentant la langue

2) l'écriture système empiriquement second de la langue

Le seul problème est celui du rapport de l'écriture et de la langue

II Analyse de l'expérience de l'acquisition de l'écriture. Problème synchronique 
III Les rapports de la langue et de l'écriture en diachronie et leur relation théorique IV La fonction sémiotique de l'écriture.

\section{Le lien «primordial » entre la langue et l'écriture : que nous apprennent les récits d'invention de l'écriture ?}

4.1. D'une part, Benveniste remarque que pour approcher l'invention de l'écriture, on peut prêter attention à la notion de message :

Une notion qui me paraît importante et qui n'est pas encore explorée dans ses rapports avec l'écriture est celle de message. Le messager récite un texte qu'il a mémorisé. Il ne parle pas. Ce n'est pas son discours qui sort de sa bouche. Il est la bouche et la langue d'un autre. Quelle situation singulière et comment n'organiserait-elle pas un discours tout particulier ! (Benveniste 2012, 98)

Relisons la légende sumérienne de la naissance de l'écriture, à laquelle Benveniste se réfère sans aucun doute dans l'extrait ci-dessus : Enmerkar et le Seigneur d'Aratta. Cette légende raconte la lutte entre le roi d'Uruk, Enmerkar, et le seigneur d'Aratta (ville sans doute située dans ce qui correspond à l'Iran), pour les faveurs de la déesse Inanna et la domination d'Uruk sur Aratta, car Enmerkar ne dispose ni de pierres, ni de métaux précieux pour honorer la déesse Inanna dans son temple. Des échanges entre les deux seigneurs ont lieu et c'est un messager qui est chargé de transmettre les messages dans lesquels Enmerkar prie le seigneur d'Aratta de reconnaître sa suzeraineté et de lui faire parvenir les richesses qu'il convoite.

S'ensuivent une série d'affrontements, qui se situent sur le plan de l'intelligence et de l'astuce. Une quatrième fois, Enmerkar envoie son messager. Mais celui-ci, au bout de quelques voyages, est fatigué et incapable de répéter le message. Voilà que la parole se révèle insuffisante. Alors Enmerkar façonne un pâton d'argile, sur lequel il écrit le texte du message, inventant du même coup l'écriture et son support.

Le seigneur de Kulab (=Enmerkar) façonna de l'argile ( $\mathrm{im}$ ) et y inscrivit un message comme on fait sur une tablette $(d u b)$. Avant cette époque, le fait d'inscrire un message sur de l'argile (im) n'existait pas. (Glassner 2000, 31)

C. Herrenschmidt explique que

l'écriture, selon la légende, naît des limites du messager : sa bouche, organe de la parole, sa parole ne suffisent plus à un discours complexe. L'écriture signifie une critique de la parole.

Et ceci réagit sur nos connaissances. Car, après tout, l'artefact du début de l'invention de l'écriture, la bulle enveloppe, ressemble à l'organe critiqué : chose ronde, creuse et contenant ce qu'elle doit faire connaître. La bulle qui contenait les calculi, ces nombres qu'elle donna ensuite à voir, évoqua sans doute une bouche qui contient la langue et les mots qu'elle peut extérioriser. (Herrenschmidt 2005, 18-19)

On le voit, l'artefact premier de l'écriture cunéiforme signifia l'extériorisation d'un organe du corps humain, organe associé au système graphique en gestation. La bouche qui dit des noms de nombres et des mots que l'on allait écrire. Il s'agit aussi d'un changement de pratique corporelle et la seconde pratique transforme de la matière (Klock-Fontanille 2014).

4.2. D'autre part, Benveniste, s'il fait allusion au récit d'invention de l'écriture (qui est en même temps l'invention du support d'écriture), ne recherche pas l'origine de l'écriture :

Je ne fais pas de génétique des écritures; je ne recherche pas l'origine de l'écriture. Je veux seulement voir quelles solutions l'homme a données au problème de la « représentation graphique », et je constate que, aussi bien dans l'antiquité la plus reculée que nous puissions atteindre que dans les temps modernes, l'homme 
commence toujours par représenter graphiquement l'objet du discours ou de la pensée, c'est-à-dire le référent. La tendance « naturelle » est de communiquer par un moyen graphique les choses dont on parle, et non le discours qui en parle. Il est donc inexact, pour qui embrasse tout l'ensemble des manifestations de l'écriture, que l'écriture soit le signe de la langue, qui est elle-même le « signe » de la "pensée ». On ne peut dire de l'écriture qu'elle est signe de signe. Elle est devenue seulement une transcription de la parole. (Benveniste 2012, 100)

Remarquons que le référent arrive ici intempestivement. Ce qui est représenté, c'est déjà une configuration sémiotique, du moins le contenu organisé d'une configuration sémiotique, qui reçoit son expression d'une inscription matérielle.

Dans la leçon 11, Benveniste développe ce point de vue et insiste sur le fait que cette découverte, cette " grande innovation » a été réalisée de façon indépendante en plusieurs points du monde et chaque fois avec des moyens différents. Il précise : «Toutes ces inventions ne sont pas les étapes d'un développement linéaire. Chacune d'elles est un commencement absolu, indépendant des autres systèmes » (Leçon 11, p. 107). Par cette insistance, Benveniste, rompt là avec la tradition évolutionniste des historiens de l'écriture (Février, Diringer, Cohen, etc.), dont S. Battestini rappelait les principes en 1997 (cf. plus haut).

4.3. Enfin, soucieux de défini clairement son objet, Benveniste interroge la relation langueécriture. C'est quelque chose qui semble aller de soi, mais cela n'a pas toujours été le cas dans le champ de la recherche. I. Fenoglio rappelle que c'est le postulat de Benveniste :

Dans sa reprise de la question de l'écriture, dans les Dernières leçons, il y a cette volonté de comprendre en quoi l'écriture s'est inscrite comme liée intrinsèquement à l'homme parlant. En quoi, plus que quand, en quoi et comment. (Fenoglio 2017, 2012).

Un détour par la question du déchiffrement des écritures permet d'apporter un éclairage intéressant à ce propos. Dans une étude sur le déchiffrement des écritures, nous avons montré que

la relation langue-écriture semble aller de soi pour nous, mais cela n'a pas toujours été le cas dans le champ de la recherche, et cela explique le fait que la question du déchiffrement soit longtemps restée bloquée. Si on prend le cas des hiéroglyphes égyptiens par exemple, il faut attendre le XVII ${ }^{\text {ème }}$ siècle pour comprendre qu'ils correspondaient à une langue ; et même une fois que la chose a été admise, il a encore fallu deux déblocages (qu'on doit, en fait, à Champollion) : d'une part, admettre que l'écriture constitue un système, et d'autre part comprendre que ce système est un code autonome par rapport a la langue, parfois même différent. (Klock-Fontanille 2018, 194)

$\mathrm{Au}$ XVII ${ }^{\mathrm{ème}}$ siècle, pour Kircher, l'un des plus grands savants de l'époque, dans son Prodromus coptus sive aegyptiacus (1636), le principe posé est que l'hiéroglyphe exige, de par sa nature symbolique, une interprétation d'intuition, incompatible avec la recherche de valeurs de convention, correspondant à des mots ou des sons du langage. Cependant, le même Kircher s'est aussi intéressé à l'écriture chinoise. Il publie en 1664 un ouvrage magistral sur la Chine, qui connaîtra un énorme succès (China monumentis qua sacris qua profanis, nec non variis Naturae et Artis Spectaculis, aliarum rerum memorabilis argumentis illustrata). Kircher compare l'écriture chinoise avec les hiéroglyphes égyptiens. Il soutient que la différence réside dans le fait qu'on a d'un côté, une écriture ordinaire qui correspond à des mots, à une langue, destinée à des offices vulgaires, et de l'autre la sublimité des hiéroglyphes égyptiens. Pour Kircher, les caractères chinois ne constituent pas un mystère à résoudre comme les hiéroglyphes égyptiens. Il s'agit d'une écriture utilisée encore de son temps dont la clé avait été largement dévoilée. Mais surtout, les caractères chinois s'épuisent dans leur correspondance avec les mots de la langue, leur interdisant tout mystère, contrairement aux 
hiéroglyphes égyptiens. Les caractères chinois entretiennent donc une relation univoque avec les mots de la langue qui révèle leur pauvreté par rapport aux hiéroglyphes égyptiens. Le caractère chinois n'a rien de mystérieux, et il ne sert pas à cacher des vérités aux profanes, c'est un instrument ordinaire de communication, en relation avec une langue, le chinois.

Il faudra attendre Champollion, au début du XIX ${ }^{\text {ème }}$ siècle, pour mettre en place la reconnaissance du rapport langue-écriture, jusque dans le système égyptien, c'est-à-dire la valorisation du langage. En effet, lorsqu'il commence à étudier la pierre de Rosette, Champollion est de plus en plus conscient de l'apport potentiel de la langue copte à la compréhension de l'égyptien. Et il se servira de cette correspondance, qui sera une des clés du déchiffrement. Cette rigueur de correspondance entre écrit et parlé, qui définit toute lecture, était à l'opposé des conceptions symbolistes. C'est ce qui a permis l'émergence de la notion de déchiffrement au début du XIX ${ }^{\text {ème }}$ siècle.

Et d'autre part, à cette valorisation du langage, il faut ajouter une autre notion qui apparaît à la même époque - et qui nous intéresse ici - : on se pose la question du statut scientifique de l'objet qu'on étudie. On commence à considérer l'écriture comme un ensemble signifiant que l'on soupçonne de posséder une organisation, une articulation interne autonome.

Donc, l'écriture est un système et c'est le déchiffrement qui le (re)construit. Deuxièmement, cette construction est le résultat de la correspondance entre la langue et l'écriture. Cela a deux conséquences : (1) si l'écriture est un système qui possède une organisation interne, on peut la soumettre à l'analyse ; (2) dire qu'il y a une correspondance entre la langue et l'écriture, c'est bien sûr dire que l'écriture transcrit la langue, mais c'est aussi dire que l'écriture est le produit de deux modes de communication hétérogènes et complémentaires, qu'elle relève à la fois du visuel et du graphique d'un côté, et du verbal de l'autre. Et c'est cela qui permettra que la correspondance, la synchronisation fonctionne.

L'écriture est " pluricode », intermédiale, et non simplement un code second destiné à représenter le code premier et principal qu'est la parole. Toute la question du déchiffrement repose sur ce postulat : il y a une relation entre langue et écriture. Il faut alors se demander comment se fait cette synchronisation, cet ajustement. Nous avons montré que cet ajustement ne pouvait pas se réduire à une traduction (parce que reposant sur une identité entre les deux codes), mais que c'est un processus de conversion qui est à l'œuvre, voire d'interprétation, c'est-à-dire de l' « assignation d'une signification à une séquence linguistique (Rastier 1987, 274).

Ce détour par la question du déchiffrement nous permet de mettre en évidence deux éléments essentiels pour la question de l'écriture dans la réflexion de Benveniste :

1. la relation langue-écriture est une conversion,

2. l'écriture est un système sémiotique

\section{5. «Comment repenser à neuf, dans leur relation primordiale, la langue et l'écriture » (p. 91)}

\subsection{Langue et écriture sont indissociables}

Les premières phrases de la leçon 8 , la première consacrée à l'écriture, mettent en avant, le caractère indissociable des deux :

Nous vivons dans la civilisation du livre, du livre lu, du livre écrit, de l'écriture et de la lecture. Notre pensée est constamment, à quelque niveau que ce soit, informée d'écriture.

Cela met en rapport de plus en plus intime, on ne peut plus intime, avec l'écriture la langue entière, la parole et la pensée même, qui ne se dissocie plus de son inscription réelle ou imaginée. Toute réflexion sur la langue, en particulier, fait surgir en notre 
pensée la forme écrite où les signes linguistiques prennent réalité visible. (Benveniste 2012, 91)

C'est la base de sa réflexion, et c'est ce qui le différencie de Saussure. Puis il fait des mises au point méthodologiques, en prenant appui sur les thèses de Saussure :

1. Pour Saussure, l'écriture est l'écriture alphabétique (il y a donc forcément un lien avec la langue) et la langue est la langue moderne. Or, ce sont des relations spécifiques, pas universelles.

2. Pour Saussure, le lien entre la langue et l'écriture est un lien de subordination : l'écriture est une représentation de la langue. Comme le rappelle J.-C. Coquet :

Benveniste part de l'écriture, terme pivot, et il remonte de l'écriture à la parole, de la parole à la langue, de la langue à la société, de la société à la culture, et [...] de la culture à la nature. (Coquet 2016, 61-62)

3. Or, il faut considérer l'écriture comme un système sémiotique, ce que Saussure ne fait pas : Si on pose que l'écriture est en soi et pour soi un système sémiotique, il faut en tirer les conséquences. La graphè « représente » la phonè, tel est le principe. Donc, rien ne peut et ne doit faire obstacle à cette représentation ni l'interpréter autrement qu'elle l'admet en soi. Il faut ici garder l'écriture comme établissant une relation réversible biunivoque entre deux termes et deux seulement : graphè $\leftrightarrow$ phonè. (Benveniste 2012, 92)

\subsection{Comment s'établit cette relation? La relation langue-écriture est une relation de conversion réciproque.}

Benveniste rompt avec la vision évolutionniste de l'écriture, avec la conception saussurienne selon laquelle l'écriture est subordonnée à la langue, dont elle ne serait qu'une sorte de calque (vision représentative de l'écriture). S'il remonte à l'invention de l'écriture, c'est pour interroger cette relation. Il constate que l'écriture a peu à peu été considérée comme un calque de la langue. Or, comme l'invention de l'écriture le montre, ainsi que la question du déchiffrement, nous sommes en présence de deux codes. C'est pourquoi, ce sont les procédures de conversion, de «transposition » (p. 94) qui vont l'intéresser : il parle à deux reprises de « la conversion soudaine de la langue en image de la langue. » (p. 93). Ou encore : « rendre intelligible le langage intérieur est une opération de conversion qui va de pair avec l'élaboration de la parole et l'acquisition de l'écriture » (p. 95). Cela lui permet d'interroger les diverses solutions de la représentation graphique de la signifiance.

\subsection{L'écriture est un système sémiotique dépendant et homologue du système de la langue.}

L'écriture est une sémiotique : les sémiotiques sont des systèmes de signes, comprenant un plan de l'expression et un plan du contenu (Hjelmslev 1966, 1968-1971, 1971). Mais leur spécificité, c'est que chacun de ces plans doit avoir une structure propre : il faut qu'on puisse dissocier une expression et un contenu; le plan de l'expression et le plan du contenu ne sont pas conformes l'un à l'autre : ils sont isotopes et hétéromorphes. Le plan de l'expression est en présupposition réciproque avec le plan du contenu et leur réunion, lors de l'acte de langage, correspond à la semiosis.

Mais l'analyse du plan d'expression et l'analyse du plan du contenu n'en sont pas moins, pour leur part, entièrement dépendantes l'une de l'autre. Hjelmslev appelle «fonction sémiotique » la similarité des analyses du plan d'expression et du plan du contenu. Cette fonction sémiotique est toujours accomplie par les textes : bien que les éléments d'expression et les éléments de contenu soient, en dernière analyse, non conformes les uns aux autres, 
chaque élément analysé dans ce texte manifeste une solidarité entre l'expression et le contenu.

Par ailleurs, rappelons que certains systèmes sémiotiques, comme l'écriture, selon notre hypothèse, sont des codes à double articulation, à savoir des codes où les plus petits éléments du plan de l'expression ne connaissent pas d'équivalents dans le plan du contenu.

Dans les codes à première articulation seulement, tous les éléments du plan de l'expression trouvent chacun un équivalent dans le plan du contenu. Dans le cas d'une écriture, l'expression se décompose en substance et en forme :

- la substance correspond à ce que Klinkenberg appelle « objet graphique ». Si cette substance est sans forme, c'est une matière.

- La forme, c'est la structure, le système ; par exemple, dans le cas de notre alphabet, il s'agit de la langue française.

La question se posera alors de savoir comment on passe de la substance à la forme. Par exemple, dans le cas d'une écriture inconnue, nous sommes en présence d'une substance de l'expression : une réalisation concrète, dont nous postulons qu'il y a un système, une organisation. Mais nous n'arrivons pas à passer de cette substance à la forme. J. Chadwick ne dit pas autre chose à propos de Ventris s'attaquant au Linéaire $b$ de Crète :

L'œil d'un architecte ne voit pas dans un bâtiment une simple façade, un assemblage d'éléments décoratifs et fonctionnels : il voit au-delà de l'apparence et sait distinguer les traits essentiels du dessin, la structure des parties, la charpente de l'ouvrage. C'est ainsi que Ventris était capable de discerner dans la confondante diversité des signes mystérieux de cette écriture les schémas et les constantes qui révélaient la structure cachée. C'est cette qualité, le don de saisir l'ordre sous l'apparence de la confusion qui est la marque des grands hommes, en tout ce qu'ils ont produit. (Chadwick 1972, 17)

C'est le cas bien connu de l'étrusque. Pour le cas de l'étrusque, on peut mettre en relation la substance de l'expression et la substance du contenu (on peut lire les textes et les comprendre). Mais on ne peut pas passer de la substance de l'expression à la forme de l'expression : quelle langue, ou plus exactement, quel système linguistique y-a-t-il derrière ?

De manière plus générale, si on propose que toute écriture est un système sémiotique, on considérera qu'elle est constituée d'une matérialité (une substance) corrélée à une forme de l'expression. Ainsi tels « objets graphiques » correspondront-ils à tel code linguistique.

Ce dernier ne correspond pas au plan du contenu : réduire l'écriture à l'expression et faire de la langue le contenu serait revenir à une vision représentative de l'écriture. Ce serait aussi considérer l'écriture comme un code à une seule articulation.

Benveniste conçoit l'écriture comme un système sémiotique. Les inventeurs, nous dit-il, «projettent dans leur écriture le type de représentation qu'ils se font de leur langue » (p. 110) ; il existe une « liaison étroite entre le type d'écriture et le type de langue, entre la manière de dissocier les éléments de la parole et la manière d'écrire ces éléments » (p. 117). C'est le guidage épilinguistique, ou plutôt ici épisémiotique. Benveniste prend plusieurs exemples :

À l'opposé de ce système unique, de cet exemple parfait du chinois où l'unité graphique est identique à l'unité du signe : chaque signe graphique coïncidant avec un signe de la langue ; il n'y a alors ni manque, ni excès ; l'unité graphique est le mot ; à l'opposé donc de ces systèmes se trouvent tous les systèmes où l'unité graphique est inférieure à l'unité linguistique, c'est-à-dire au signe. Dans ces systèmes l'unité graphique est une partie du signe (par exemple la syllabe).

La coupe syllabique de la parole est, me semble-t-il, la coupe naturelle, car on ne peut isoler d'un support vocalique un son quel qu'il soit. L'unité de décomposition de la parole sera donc ou une voyelle ou un segment incluant une voyelle (CV ou VC). L'articulation naturelle de la parole est reproduite comme articulation naturelle de l'écriture. (Benveniste 2012, 109) 
Dans les langues sémitiques (comme l'arabe ou l'hébreu) les consonnes priment sur les voyelles et les racines tri-consonantiques portent le sémantique de la langue. La vocalisation, au départ, n'existe pas. Dans l'alphabet grec, au contraire, il y a un statut identique pour les consonnes et les voyelles et la voyelle est essentielle pour déterminer le sens de l'unité.

Avec l'alphabet, l'articulation naturelle de la parole est reproduite comme articulation naturelle de l'écriture.

Nous voyons que la thèse de Benveniste se précise : la relation de l'écriture est bien une relation de conversion réciproque : celui de la conversion du discours en forme linguistique (il faut ramener l'énoncé à ses parties constituantes et reconnaître qu'il y a un nombre de signes limité) et celui de l'écriture comme système formel (Leçon 11, p. 111) : la substance devient forme. Mais c'est l'invention de l'écriture qui pousse les sociétés à prendre conscience de l'existence d'un système linguistique, ce que nous appelons le linguistique ; en cherchant à fixer les messages et la parole, les humains inventent la langue. On pourrait dire que l'expérience d'une formalisation de l'expression et du contenu scripturaux sert de support à la transposition du même type d'analyse à la langue.

Résumons : l'écriture, dit Benveniste, est « relais de la parole » ou « forme secondaire de la parole » (p. 131), c'est un système parallèle à celui de la langue, résultant d'une conversion d'un code dans un autre code : «c'est de la parole transférée de l'ouïe à la vue : la parole, seulement auditive, devient l'écriture, seulement visuelle » (p. 131). "Le système graphique reproduit la langue ». De fait, «la langue et l'écriture signifient exactement de la même manière » (p. 127) et " l'écriture alphabétique est l'instrument qui a permis à la langue de se sémiotiser elle-même ». Cela veut dire que le parlant s'arrête sur la langue », objectivée par les représentations graphiques, « au lieu de s'arrêter sur les choses énoncées » (p. 113).

Benveniste en déduit que la langue est le seul système signifiant qui puisse se décrire luimême et pour cette objectivation un instrument méta-linguistique est nécessaire : c'est l'écriture. Coquet commente en disant qu'elle est «l'interprétant de la langue » (Coquet 2016, 63).

Par conséquent, le même mouvement qui met peu à peu en place cette relation biunivoque dont il parlait, déclenche la formalisation entendue comme un procédé qui permet de détacher la langue de son utilisation. Ce sont les processus de décontextualisation et d'objectivation, qui font partie des fonctions essentielles de l'écriture, que Benveniste a évoqués dans la leçon 8. Ces processus seront repensés par Auroux, et Goody, en particulier.

\section{Représentation iconique et représentation linguistique : les implications des propositions de Benveniste.}

L'écriture non seulement organise la langue, mais elle la matérialise avec l'image de la langue : "La langue est soudain convertie en une image de la langue », écrit Benveniste (p. 93). Comme l'explique I. Fenoglio:

Benveniste affirme que l'écriture fait prendre conscience de l'existence de la langue, elle la matérialise avec l'image de la langue : «La langue est soudain convertie en une image de la langue » (BENVENISTE, 2012, p. 93), « convertie en une image » et non pas « simple image trompeuse détachée ». (Fenoglio 2017, 225)

L'approche de Benveniste est bien une approche linguistique certes, mais pas strictement logocentrique, au sens traditionnel du terme. Chez lui, l'écriture ne se réduit pas à ses fonctions glossiques.

Un moment nouveau dans la pensée de Benveniste prend son départ ici. Ce qui l'intéresse, c'est de comprendre comment l'iconisation déclenche et affine la formalisation de la langue intérieure. L'écriture (leçon 12) est un système de signes qui ressemble beaucoup plus au langage intérieur qu'à la chaîne du discours. Voici ce que dit Benveniste : 
Saussure défend l'idée banale de l'écriture comme système subordonné à la langue. Or rien n'empêche d'imaginer un « signe iconique » (ou « symbolique », comme on voudra, le choix des termes est tout à fait indépendant de la terminologie de Peirce) qui associerait la pensée à une matérialisation graphique, parallèlement au «signe linguistique » associant la pensée à sa verbalisation idiomatique. La représentation iconique se développerait parallèlement à la représentation linguistique et non en subordination à la forme linguistique.

Cette iconisation de la pensée supposerait probablement une relation d'une autre espèce entre la pensée et l'icône qu'entre la pensée et la parole, une relation moins littérale, plus globale. (Benveniste 2012, 95)

Voici la réflexion que ce passage suggère à Coquet :

La fonction du langage est de «re-reproduire la réalité ». [...] Le langage iconique re-présente le vécu, re-produit l'émotion. [...] L'une des propriétés du langage iconique est donc qu'il ne communique pas. La fonction de tout langage, on le sait est d'abord de signifier, mais seul le langage ordinaire associe signification et communication, dans cet ordre. Centré sur le corps, le langage iconique compose un univers second [...]. Les icônes en se substituant aux signes conceptuels du langage ordinaire traduisent le passage des choses d'un univers à l'autre. (Coquet 2012)

Au fond, ce que propose Benveniste, c'est de conjurer ce que Orly Goldwasser appelait « la peur d'Horapollon » à propos de l'évolution des études sur l'écriture hiéroglyphique égyptienne :

Durant de nombreuses années, la science égyptologique moderne a souffert de ce qu'on pourrait appeler « la peur d'Horapollon ». Hantée par « l'attitude symbolique » d'Horapollon vis-à-vis des hiéroglyphes et par l'échec spectaculaire des tentatives de déchiffrage menées sous son influence à la Renaissance, l'égyptologie moderne a tourné le dos à toute approche mêlant la sémiologie à l'étude des hiéroglyphes. Presque toutes les introductions et études portant sur le système hiéroglyphique au $\mathrm{XX}^{\mathrm{e}}$ siècle ont présenté le signe hiéroglyphique soit en tant que logogramme soit en tant que phonogramme. Une fois baptisé phonogramme, le signe est dépouillé de toute signification iconique. (Goldwasser 2009, 339)

Coquet, répondant à Pascal Quignard :

Benveniste présente, n'oppose pas, mais présente deux modes d'être. L'un qui tient aux yeux, à la vision, à l'image, et l'autre qui tient à la main, c'est l'écriture. Mais ces deux modes d'être ont ceci de particulier que ce qu'il suppose de représenté dont vous parliez tout à l'heure s'écrit aussi chez Benveniste de deux façons : re-présenter, c'està-dire présenter une deuxième fois, et puis représenter, en seul bloc, qui est une opération cognitive, intellectuelle. Donc, l'image est une présentation nouvelle d'un événement qui a eu lieu et qui suppose une continuité. C'est-à-dire que l'image ne parle pas à elle-même, alors que l'écriture, le sillon de l'écriture, trace à elle-même. Le monde est clos. Alors que l'image, ce qui dépend des yeux, est un paysage fuyant. Donc, lorsque Benveniste montre ce que lui croit être un des éléments principaux de l'image et de l'écriture d'abord par la présentation par image d'abord des événements comme chez les Esquimaux, l'image à elle-même, en elle-même n'est jamais suffisante. Il faut qu'il y ait une suite. Donc, il y a bien récit, mais c'est un récit à voir, pas un récit à conceptualiser. C'est un récit qui suppose une présentation nouvelle d'un événement qui doit être dans une continuité. Alors que dans ce qui dépend de la main, c'est-à-dire l'écriture, on a des traces, des sillons qui permettent de s'arrêter et qui ne demandent pas du tout de suite. On peut, et c'est là aussi l'avantage des idéogrammes chinois, on peut méditer indéfiniment sur des tracés. Donc je crois que les deux modes d'être sont à retenir dans la proposition de Benveniste. (Coquet 2016, 323-324) 
Nuançons ces propos. Faut-il y voir une référence à Lessing qui, dans son Laocoon (1766), a canonisé la dichotomie entre langue et image, prononçant le divorce entre dire et montrer, représentation et présentation, discursif et iconique ? Notons que l'image est aussi produite par une technique, parfois même par la main. Notons aussi que, à la base de toute écriture il y a l'image, puisque le dessin et l'écriture sont l'un et l'autre des signes visuels. Il y a un fond commun. Si, au moment de l'invention de l'écriture, le travail formel de fabrication des signes est nécessaire, il ne peut, cependant, se suffire à lui-même. Il ne peut être dissocié de l'aspect sémantique, car ce n'est pas la "substance » (L. Hjelmslev) des signes qui importe, mais leur valeur. Les préoccupations de sens sont omniprésentes dans les procédures de création. Rendre les signes signifiants, cela signifie deux choses :

(i) renvoyer à un signifié linguistique : les signes traduisent des mots de la langue, ils sont donc autant de logogrammes. Les signes ne sont pas des outils mnémotechniques pour une représentation mentale, ils sont utilisés pour dénoter un morphème linguistique.

(ii) Ces signes sont à la base des images dont ils ont gardé un certain nombre de propriétés. L'utilisation de ces signes permet certes un lien avec un signifié linguistique, mais aussi avec un contenu non linguistique. Depuis E. Laroche, en passant par M. Marazzi, P. Vernus et d'autres, de nombreuses études ont montré la fonction idéologique de l'écriture hiéroglyphique, fonction qu'elle a pu développer grâce à ses propriétés intrinsèques. Pensons aux travaux que Vernus a menés sur les potentialités du système hiéroglyphique égyptien (Vernus 1982, 1985, 1988) ou aux études menées sur les hiéroglyphes anatoliens. C'est une évidence de dire que si les Hittites, qui avaient déjà à leur disposition une écriture - l'écriture cunéiforme -, ont inventé un nouveau système d'écriture - un système hiéroglyphique, fondé sur l'image -, ce n'est pas pour qu'il redouble celui qui existait (Laroche 1963 ; KlockFontanille 1998). L'écriture a investi le monde de l'image et l'a doté de fonctions sémiologiques qu'il ignorait auparavant. Il est donc nécessaire de distinguer entre l'identité d'un dessin en tant que marque visuelle et spatiale et la valeur qu'il acquiert au moment d'être intégré dans le système d'écriture (Vernus 2016).

J.-J. Glassner explique qu'il existe deux types de représentations picturales,

l'un qui n'a besoin d'aucune justification linguistique, le second qui est accroché à la langue. L'écriture appartient au second, elle qui se situe précisément à l'inter- section de ces deux plans de l'activité cognitive que sont l'image et la parole, et qui relève de manière définitoire de la linguistique et de la sémiologie, se distinguant ainsi de toutes les autres formes d'expression visuelle. (Glassner 2009)

L'écriture assume donc deux familles de fonctions : (1) des fonctions glossiques; dans ce premier cas, les signes scripturaux ont pour plan de l'expression des fonctions ou des données $\mathrm{du}$ code linguistique (ce sont ces seules fonctions qui, d'habitude, retiennent le linguiste tenant de la conception stricte de l'écriture); et (2) des fonctions grammatologiques : dans ce second cas, les signes graphiques ne renvoient pas à des fonctions du code linguistique mais assument une autre fonction sémiotique. En d'autres termes, à partir d'une même inscription, deux perceptions concurrentes contribuent ensemble au dégagement de valeurs sémiotiques (linguistique $v s$ iconique), et on peut montrer comment s'articule la tension entre deux valences perceptives : d'un côté la «reconnaissance linguistique », et de l'autre l' « identification iconique ».

L'écriture peut alors être considérée comme une sémiotique reliée à d'autres sémiotiques. Les signes graphiques peuvent renvoyer aussi bien à un signifié linguistique et/ou non linguistique (idéologique, pratique...), ou les deux à la fois. En d'autres termes, l'écriture, en tant que sémiotique graphique, est un déclencheur de transposition intersémiotique, du graphisme vers une ou plusieurs des pratiques du langage verbal, ainsi que vers d'autres pratiques associées (rituelles, par exemple).

Comme on l'a vu, Benveniste propose de renouveler les problèmes des relations entre la langue et l'écriture en posant ce principe fondamental: l'écriture est une forme secondaire/seconde de la parole (p. 131) : c'est la parole transférée de l'ouïe à la vue : la 
parole, seulement auditive, devient l'écriture, seulement visuelle. Elle permet à la langue de se sémiotiser elle-même. On a toute une série de formules : l'écriture est de la «langue visible », c'est de la "parole même fixée dans un système secondaire de signes », la «parole convertie par la main en signes parlants », « la main prolonge la parole », " Entre la bouche et l'oreille, le lien est la phonie émise-entendue ; entre la main (l'inscription) et l'œil, le lien est la graphie tracée-lue ».

\section{Des continuateurs?}

Cette relative autonomie de l'écriture ne remet pas fondamentalement en question le principe d'interdépendance entre la langue et l'écriture, mais elle contribue à étendre le domaine de l'écriture. C'est ce que propose Klinkenberg, par exemple, qui plaide « pour une conception médiane où sont garantis 1) le caractère glossique de l'écriture et 2) sa relative autonomie par rapport à la langue orale » (Klinkenberg 2005, 163) :

L'écriture est un code sémiotique dont le plan de l'expression est constitué d'unités discrètes combinables et ordonnées dans un espace perceptible, et dont le plan du contenu comprend des unités linguistiques combinables. (Klinkenberg 2005, 163)

Cette définition illustre la position médiane qui celle de Klinkenberg : elle met en avant la relative autonomie des écritures, mais surtout, ce qui est pour lui le fondement de l'écriture, à savoir la spatialité : « La relative autonomie de l'écriture prend sa source dans la spatialité », explique-t-il (Klinkenberg 2005, 165) :

La part la plus importante des spécificités de l'écriture lui vient du double fait qu'elle est articulée à la langue - laquelle présente un caractère de linéarité - tout en présentant un caractère spatial, caractère essentiel qu'elle tient à l'origine, de son tropisme pour le canal visuel. (Klinkenberg 2005, 160-161)

Quant à Roy Harris, à la fois lecteur et critique de Saussure, comme l'a été Benveniste, il part de l'analyse de la conception erronée de l'écriture comme notation de la parole qui a, selon lui, contaminé la linguistique pour proposer une approche intégrationnelle (Harris 1986, 1993, 1998). Celle-ci donne sa place à l'écriture au sein d'une sémiologie revisitée, qui n'est ni phonocentriste, ni phonographiste, ni même autonomiste. Il ne s'agit pas de dénoncer le postulat représentatif, celui qui annexe l'écrit à la parole, mais de mettre en place la sémiologie propre de l'écriture, en repérant ses traits spécifiques et son fonctionnement. Comme Benveniste, Harris se tourne vers la question de l'origine de l'écriture, objet de l'ouvrage The Origin of Writing, cherchant ainsi, sinon à résoudre la question même de l'origine de l'écriture, du moins à poser un certain nombre de questions et "déconstruire » (pour reprendre un terme derridien) un certain nombre de préjugés qu'on trouve aussi bien chez les linguistes que chez les sémioticiens.

Comme d'autres, Harris revient sur deux préjugés : (1) l'écriture comme retranscription de la parole (selon Harris, «dans la civilisation occidentale, l'écriture et la parole ont été pendant des siècles enfermés dans une relation symbiotique par essence », ce qui a faussé notre approche de l'écriture) ; (2) la tyrannie de l'alphabet. C'est ce qu'il nomme « l'illusion évolutionniste » qui consiste à voir l'histoire de l'écriture comme une marche progressive et quasi inéluctable vers la représentation de la parole (Klock-Fontanille et Pagès 2016).

Contrairement à Benveniste, il pense que l'origine et l'avenir de l'écriture présentent des liens auxquels la parole est tout à fait étrangère. En d'autres termes, l'écriture n'est pas une représentation graphique de la parole mais un système de représentation des idées qui possède son autonomie. Une notation graphique peut véhiculer du sens indépendamment de la représentation d'un mot de la langue parlée.

Klinkenberg présente de la manière suivante la définition de l'écriture selon Roy Harris : Une écriture est faite de signes 1) présentant un caractère discret et 2) se combinant de manière répétitive dans une structure spatiale fixe (le segment de ligne droite par 
exemple, ou une spirale, comme dans le disque de Phaestos). Tout système de signes présentant cette double caractéristique est alors dit écriture. (Klinkenberg 2005, 162)

Mais Harris, prônant une théorie intégrationnelle, rappelle que se demander ce qu'est l'écriture est une mauvaise question. Il préfère se demander « quelle sont les activités qui, tout en dépendant de l'écriture, sont présupposées par l'écriture même » (Harris 1998, 15). Car, précise l'auteur,

l'écriture elle-même présuppose certaines activités sans lesquelles elle serait une impossibilité. Il s'agit, précisons-le, non seulement d'activités pragmatiques mais aussi d'activités mentales ou cognitives exercées dans le cadre d'une situation sociale. (Harris 1998, 15)

Selon R. Harris, l'écriture n'existerait pas sans toute une intégration d'activités dont elle fournit un moyen technologique essentiel :

Pour l'intégrationniste, il faut remplacer ce genre d'abstraction par une théorie du signe où la signification naît - et renaît constamment - d'une intégration d'activités dans un contexte spécifique. (Harris 1998, 16)

Pour l'auteur, un élément essentiel de son approche de l'écriture, c'est la notion d'espace : l'écriture n'est, selon lui, rien d'autre qu'un moyen d'utiliser l'espace pour les besoins de la communication

Approche linguistique? Approche sémiotique? D'un côté, Harris se souvient que la linguistique avait écarté l'écriture de son objet, et qu'un de ses maîtres parmi les plus influents, Saussure, l'avait confiée à la «sémiologie ». Mais d'un autre côté, Harris ne dénonce pas tant la conception représentative de l'écriture, que la doxa qui en est issue : c'est la réduction au principe « une lettre : un son », c'est d'avoir traité la représentation de la parole comme critère pour reconnaître l'émergence de l'écriture. Ce qui est remarquable chez Harris, c'est que tout en fondant sa sémiologie intégrationnelle sur une critique de Saussure, il lui est resté profondément fidèle par son approche linguistique.

\section{Conclusion : Quelques pistes pour un nouveau point de vue sur le problème.}

Nous terminons cette réflexion suscitée par la lecture de Benveniste par deux questions et une proposition qui consiste à adopter un point de vue global.

Première question : pourquoi le point de vue sémiotique détache-t-il le phénomène scriptural de la langue ? Parce qu'il le replace dans un autre ensemble de productions humaines symboliques (toute forme de productions sémiotiques, dont l'inscription n'est qu'une sous-catégorie, et dont l'inscription de la langue n'est qu'une sous-sous-catégorie). Plus précisément, le point de vue sémiotique, par la prise en compte de la nature iconique de l'écriture (pensons au concept de « Schriftbildlichkeit » de Sybille Krämer [Krämer, 2003a, 2003b, 2016]), propose un changement de perspective, allant d'une conception de l'écriture orientée sur la langue vers une conception de l'écriture phonémiquement neutre, «à la lumière de laquelle l'esthésie, la matérialité et l'opérativité des écritures sont examinées comme des ressources productives des activités théoriques, artistiques et pratiques » (Krämer 2016).

Deuxième question : pourquoi le positionnement de l'écriture dans cet ensemble de productions sémiotiques fait-il basculer le problème du côté anthropologique ? En d'autres termes, pourquoi l'écriture, appartenant et interagissant avec d'autres types d'expression symboliques, s'est ainsi aussi fortement associée à l'expression verbale ? Deux réponses :

1. Parce que les écritures deviennent des productions culturelles porteuses d'identités culturelles différentes des langues, et le plus souvent de plus large périmètre. Des anthropologues, comme Marazzi, Perri, Cardona, ont mis en évidence que l'acte scriptural et ses mécanismes ne reflètent pas seulement le besoin de transcrire glossiquement une langue, 
mais aussi un ensemble de valeurs culturelles, de connaissances encyclopédiques, de manifestations idéologiques et de finalités économiques et administratives spécialisées (Marazzi 2010 ; Cardon 1981 ; Perri 2007, 2014). Les exemples sont nombreux, qu'ils soient anciens (Egypte, Anatolie, Crète, Maya) ou modernes (Mustapha Kemal Atatürk et l'alphabet latin, pour citer un exemple célèbre).

2. Parce qu'au sein de cet ensemble, les questions qui se posent sont non seulement celles du "mode de signifier » de chaque sous-catégorie (notamment le rapport entre l'écriture et la langue), mais aussi et surtout la nature des pratiques culturelles qui les produisent (modeler, tailler, inscrire, archiver, transmettre à distance, protéger, commémorer, etc.). Il apparait alors que parmi toutes les pratiques de productions symboliques (pratiques gestuelles, pratiques de transformation de la matière, pratiques d'édification et de constructions d'espaces, pratiques d'émissions de sons corporels, etc.), l'écriture n'appartient pas au même genre anthropologique que la langue. C'est ce que nous apprend la légende d'Enmerkar que nous avons citée plus haut. La légende dit que, après quatre voyages, le messager d'Enmerkar est fatigué et incapable de répéter le message. Comme la parole se révèle insuffisante, Enmerkar façonne un pâton d'argile, sur lequel il écrit le texte du message, On voit bien ici que la question de la distance n'est pas que circonstancielle : elle installe deux zones anthropiques différentes, qui conditionnent l'usage de deux pratiques différentes. Quelque chose comme la différence entre la communication interpersonnelle directe et la transmission à distance. Relisons le commentaire de Glassner :

La stratégie d'Enmerkar est limpide. La première sommation exceptée, il cherche invariablement à faire en sorte que son adversaire se saisisse d'un objet qu'il lui fait porter. A deux reprises, cet objet est un sceptre, symbole ostentatoire de son autorité et de son pouvoir. En d'autres termes, il lui tend ostensiblement le même piège qui consiste à lui faire brandir un objet qui est la marque patente de sa défaite. Par eux fois, le seigneur d'Aratta sait s'esquiver. Mais, déterminé à la victoire, le roi d'Uruk invente un nouveau mode de transmission du message au moyen de l'écriture notée sur un support. Pour en prendre connaissance, le seigneur d'Aratta ne peut se dérober, condamné qu'il est à prendre dans ses mains le pâton d'argile pour lire le message dont il est le porteur.

Le texte insiste, en effet, à plusieurs reprises sur le fait qu'il doit « regarder » le message qui lui est destiné et non point l'entendre de la bouche du messager. (Glassner 2000, 29)

Le nœud du récit tient dans les cinq vers suivants :

Le seigneur d'Aratta prit/reçut du messager le tison qui lui était destiné. Le seigneur d'Aratta regarda l'argile : la parole dite, c'était "le clou est enfoncé », c'était un commandement impérieux. Le seigneur d'Aratta demeura dans la contemplation du tison qui lui était destiné.

Ces vers ont été admirablement analysés par J.-J. Glassner (Glassner 2000, 34-41). Il existe une pratique en Mésopotamie autour du transfert de propriété (cession immobilière ou foncière, aliénation d'une terre, vente d'une maison ou d'un esclave) qui consiste, lors de la conclusion d'une transaction, à enfoncer un clou en argile dans un support (par exemple, dans un mur de l'objet même de la cession); ce clou enfoncé est un marqueur de territoire qui se distingue du bornage (Glassner 2000, 38). Dans notre légende, la ruse du seigneur d'Uruk jouait d'une situation qu'il créait de toutes pièces, obligeant son compétiteur à prendre en main le support du message afin d'en saisir le sens et de le lire. Le seigneur d'Aratta tenant dans ses mains la tablette sur laquelle figure l'expression " le clou est enfoncé » et disant cette expression, tout se passe comme si le clou, marque d'appropriation, était planté au cœur de son royaume, comme si la transaction signalant le transfert de bien était conclue et qu'il n'existait plus aucune possibilité pour s'y soustraire ou l'annuler.

On le voit, l'écriture n'est pas qu'un ersatz de la parole. Le transfert de la parole vers l'écriture signifie un changement de pratique. Mais on n'est pas dans le même régime 
anthropique : le message du messager n'est pas porteur de la souveraineté, alors que la tablette l'est. Le transfert entre ces deux types de productions symboliques que sont la parole et écriture peut se faire, parce que tous deux reposent sur des régimes de croyances proches. Il en est de même entre l'image en tant que marque visuelle et spatiale et l'image qui acquiert une autre valeur lorsqu'elle est intégrée dans le système d'écriture. Ce qui passe par l'écriture et la parole passe par le même type de contraintes. C'est un transfert de substance. Ce qu'il y a de commun et ce qu'il y a de spécifique peut s'étudier à travers l'utilisation des potentialités iconiques des signes hiéroglyphiques (par exemple, dans l'écriture hiéroglyphique égyptienne ou anatolienne) et/ou des potentialités scripturales des images. Le problème global qui se pose est donc celui de la traduction intersémiotique entre deux médias.

\section{Références bibliographiques}

Battestini, S. (1997). Ecriture et texte, contribution africaine. Laval : Presses de l'Université Laval. Battestini, S. (éd.) (2006). De l'écrit africain à l'oral. Le phénomène graphique africain. Paris : L'Harmattan.

Benveniste, E. (1969). Annuaire du Collège de France, 69ème année. Paris : Imprimerie Nationale.

Benveniste, E. (1974). Sémiologie de la langue. in Problèmes de linguistique générale 2. Paris : Gallimard, 1974, 43-66.

Buyssens, E. (1943). Les langages et le discours. Bruxelles : Office de publicité.

Cardona, G. (1981). Antropologia della scrittura. Torino : Loescher.

Chadwick, J. (1972). Le déchiffrement du Linéaire B. Aux origines de la langue grecque. Paris : Gallimard.

Chiss J.-L. et Puech, Ch. (1988). Le Cours de Linguistique générale et la «représentation » de la langue par l'écriture. in Pour une théorie de la langue écrite, N. Catach (éd.), Paris : Editions du CNRS, 47-55.

Cohen, M. (1958). La grande invention de l'écriture et son évolution, Paris : Imprimerie nationale et Librairie Klincksieck.

Coquet, J.-Cl. (2012). Quelques remarques sur le langage iconique. Semen, 33, URL : http://journals.openedition.org/semen/9497

Coquet, J.-Cl. (2016). A propos de l'écriture dans la phénoménologie du langage : Benveniste, Merleau-Ponty et quelques autres. in Autour de Benveniste. Irène Fenoglio, Jean-Claude Coquet, Julia Kristeva, Charles Malamoud et Pascal Quignard (éd.), Paris : Seuil, 59-86.

Coquet, J.-Cl. et Fenoglio, I. (2012). Dernières leçons, Collège de France, 1968 et 1969. Paris : EHESS/Seuil/Gallimard.

Derrida, J. (1967). De la grammatologie, Paris : Minuit.

Dosse, F. (1992). Histoire du structuralisme. II Le chant du cygne, 1967 à nos jours. Paris : La Découverte.

Fenoglio, I. (2013). Les dernières leçons d'Emile Benveniste au collège de France. Nouveau regard sur l'écriture. Le français aujourd'hui, 181, 131-142.

Fenoglio, I. (2016). L'écriture au fondement d'une civilisation laïque. in Autour de Benveniste. Irène Fenoglio, Jean-Claude Coquet, Julia Kristeva, Charles Malamoud et Pascal Quignard (éd.), Paris : Seuil, 153-236.

Fenoglio, I. (2017). La langue et l'écriture : un écart théorique entre Benveniste et Saussure. Revista do GELNE, Natal/RN, 19, 211-236.

Fevrier, J. (1959). Histoire de l'écriture. Paris : Payot.

Gelb, I.J. (1952). A study of writing, Chicago/Londres : The University of Chicago Press [(1973). Pour une théorie de l'écriture. Paris : Flammarion].

Glassner, J.-J. (2000). Écrire à Sumer. L'invention du cunéiforme. Paris : Seuil.

Glassner, J.-J. (2009). Essai pour une définition des écritures. L'Homme, 192, URL: http://journals.openedition.org/lhomme/22268

Goldwasser, O. (2009). La force de l'icône - le « signifié élu », in Image et conception du monde dans les écritures figuratives. N. Beaux, B. Pottier \& N. Grimal (éd.), Paris : AIBL-Soleb, 336-363.

Harris, R. (1986). The Origin of Writing. Londres : Duckworth.

Harris, R. (1993). Sémiologie de l'écriture. Paris : CNRS Editions. 
Harris, R. (1998). Théorie de l'écriture : une approche intégrationnelle. in Propriétés de l'écriture. J. G. Lapacherie (éd). Op.Cit., 10, 15-17.

Herrenschmidt, Cl. (2005). Deux cas de fusion entre support et surface. in M. Arabyan et I. KlockFontanille (éd.), L'écriture entre support et surface. Paris : L'Harmattan, 15-28.

Hjelmslev, L. (1966). Le langage. Paris : Minuit.

Hjelmslev, L. (1968-1971). Prolégomènes à une théorie du langage. Paris : Minuit.

Hjelmslev, L. (1971). Essais linguistiques. Paris : Minuit.

Klinkenberg, J.-M. (2005). Vers une typologie générale des fonctions de l'écriture. De la linéarité à la spatialité. Bulletin de la Classe des Lettres, Académie Royale de Belgique, 1-6, 157-196.

Klock-Fontanille, I. (1998). Digraphie, emprunts et approximations : le problème de l'écriture dans l'empire hittite. in Propriétés de l'écriture. J. G. Lapacherie (éd). Op.Cit., 10, 53-62.

Klock-Fontanille, I. (2014). Penser l'écriture : corps, supports et pratiques. Communication \& langages, 182, 29-43.

Klock-Fontanille, I. (2016). Repenser l'écriture. Pour une grammatologie intégrationnelle. Actes Sémiotiques, 119 URL : https://www.unilim.fr/actes-semiotiques/5623

Klock-Fontanille, I. (2018). Lorsque la langue ne rencontre pas l'écriture : la question du déchiffrement des écritures. in Signatures. (Essais en) Sémiotique de l'écriture. Signata, 9, 193-212.

Klock-Fontanille, I. (2019). La recherche sur les écritures: un dialogue entre linguistique, sémiotique et anthropologie. in J. Fontanille \& A. Zinna (éd.), Dialogue entre la sémiotique structurale et les sciences, Hommage à A.J. Greimas. Langages, 213, 29-42.

Klock-Fontanille, I. et Pagès, J.-L. (2016). L'origine de l'écriture selon Roy Harris. Actes Sémiotiques, 119, URL : https://www.unilim.fr/actes-semiotiques/5624

Krämer, S. (2003b). Notational Iconicity, Calculus: On Writing as a Cultural Technik. MLN, 118/3, 518-537.

Krämer, S. (2016). Entre discursivité et iconicité, un nouveau regard sur les écritures. Actes Sémiotiques, 119, URL : https://www.unilim.fr/actes-semiotiques/5628.

Krämer, S. (2003a). « Schriftbildlichkeit » oder: Über eine (fast) vergessene Dimension der Schrift. in H. Bredekemp et S. Krämer, Bildschrift - Zahl. München : Fink Verlag, 157-176.

Laroche, E. (1963). Les Hittites, peuple à double écriture. in L'écriture et la psychologie des peuples. Paris : Armand Colin, 103-116.

Marazzi, M. (2010). Scrittura, percezione e Cultura : Qualche Riflessione sull'Anatolia in Età Hittita. Kaskal, 7, 219-255.

Perri, A. (2007). Evento linguistico vs evento scrittorio : verso un nuovo modello. Rivista di psicolinguistica applicata, VII, 2, 125-145.

Perri, A. (2014). Why writing is not (only) transcribing? Writing codes in contact: steps towards multigraphic literacy practices. Testo e Senso, 15, 75-98.

Rastier, F. (1987). Sémantique interprétative. Paris : PUF.

Saussure, F. de (1986). Cours de linguistique générale. Paris : Payot.

Testenoire, P.-Y. (2017). « La langue est une institution SANS ANALOGUE (si l'on y joint l'écriture) » : l'écriture comme problèmes dans la réflexion théorique de Saussure. Semiotica, 217, 117 133.

Testenoire, P.-Y. (2018). Sulla lettura e la scrittura : ciò che Benveniste ha letto in Saussure, e ciò che non ha letto. in Studi in onore di Daniele Gambarara, a cura di M. W. Bruno, D. Chiricò, F. Cimatti, G. Cosenza, A. De Marco, E. Fadda, G. Lo Feudo, M. Mazzeo, C. Stancati, Milan : Mimesis, 475-490 [Version française : Sur la lecture et sur l'écriture : ce que Benveniste a lu et n'a pas lu chez Saussure. Communication faite au $2^{\circ}$ Colóquio Internacional Ferdinand de Saussure : publicaçao do CLG e herença organisé par Hozanete Lima et Clemilton Pinheiro à l'Universidade Federal do Rio Grande do Norte de Natal (Brésil) en septembre 2016].

Touratier, Ch. (2004). Les écritures, approche linguistique. in Ecriture, approches en sciences cognitives, A. Piolat (éd.), Publications de l'Université de Provence, 69-90.

Vernus, P. (1982). Espace et idéologie dans l'écriture égyptienne. in Ecritures, systèmes idéographiques et pratiques expressives. Paris : Le Sycomore, 101-114.

Vernus, P. (1985). Des relations entre textes et représentations dans l'Egypte Pharaonique. in Ecritures II. A.-M. Christin (éd.), Paris : Le Sycomore, 45-69.

Vernus, P. (1988). L'ambivalence du signe graphique dans l'écriture hiéroglyphique. in Ecritures III, Espaces de la lecture. A.-M. Christin (éd.), Paris : Retz, 60-65.

Vernus, P. (2016). De l'image au signe d'écriture, du signe d'écriture à l'image, de l'image au signe d'écriture. Actes Sémiotiques, 119, URL : https://www.unilim.fr/actes-semiotiques/5633 